\title{
Cellular automaton models and traffic flow
}

\author{
A. Schadschneiderf̊ and M. Schreckenberg巳?
}

\author{
Institut für Theoretische Physik \\ Universität zu Köln \\ D-50937 Köln, F.R.G.
}

\begin{abstract}
A recently introduced cellular automaton model for the description of traffic flow is investigated. It generalises asymmetric exclusion models which have attracted a lot of interest in the past. We calculate the so-called fundamental diagram (flow vs. density) for parallel dynamics using an improved mean-field approximation which takes into account short-range correlations. For maximum velocity 1 we find that the simplest non-trivial of these approximations gives already the exact result. For higher velocities our results are in excellent agreement with numerical data.
\end{abstract}

\footnotetext{
${ }^{2}$ email: as@thp.uni-koeln.de

${ }^{3}$ email: schreck@thp.uni-koeln.de
} 
The investigation of traffic flow in the past was based mainly on the use of fluiddynamical methods. In recent years also methods of nonlinear dynamics were applied. On the other hand, due to their computational simplicity, lattice gas automata [1] were succesfully applied to simulate fluids [2] and traffic in one [3] and two dimensions [4, 5]. Similar models have also been used for the description of asymmetric exclusion processes [6]-[14] and surface roughening [15]. Several exact solutions have been obtained for asymmetric exclusion processes where the particles can move at most one lattice spacing per update step. In a more general situation one considers particles which can move over larger distances. All these models may be interpreted as discrete models for the simulation of traffic flow. Starting from realistic traffic one usually has a whole spectrum of car velocities and thus it is straightforward to introduce these more general exclusion models which then are more appropriate for comparison with 'experiments' (i.e. measurements on freeway traffic [16]).

In this letter we will consider single-lane traffic on a ring geometry (length $L$, periodic boundary conditions). Generalizations to multi-lane traffic and other boundary conditions (e.g. the bottleneck situation where one considers open boundaries) will also be discussed briefly.

The exact definiton of the model following [3] is as follows:

On a ring with $L$ sites every site can either be empty or occupied by one vehicle with velocity $v=0,1, \ldots, v_{\max }$. At each discrete time-step $t \rightarrow t+1$ an arbitary arrangement of $N$ cars is updated according to the following rules:

1) Acceleration: If the velocity $v$ of a vehicle is lower than $v_{\max }$ the speed is advanced by one $\left[v_{1}=v+1\right]$.

2) Slowing down (due to other cars): If the distance $d$ to the next car ahead is not larger than $v_{1}\left(d \leq v_{1}\right)$ the speed is reduced to $d-1\left[v_{2}=d-1\right]$.

3) Randomization: With probability $p$, the velocity of a vehicle (if greater than zero) is decreased by one $\left[v_{3}=v_{2}-1\right]$.

4) Car motion: Each vehicle is advanced $v=v_{3}$ sites.

These rules can be applied to all cars in parallel (parallel update), sequentially to randomly chosen cars (random-sequential update) or in parallel to all cars on even and odd lattice sites (sublattice-update) The rules ensure that the total number $N$ of cars is conserved under the dynamics (this is not true in the bottleneck-situation). Note that even for parallel update the randomization yields non-deterministic behaviour. For random-sequential update the probability $p>0$ is not essential because it only rescales the time axis [3]. In the following we will concentrate on the cases $v_{\max }=1,2$ and parallel update.

In the simplest case $v_{\max }=1$ the cars are allowed to move only one step during

\footnotetext{
${ }^{4}$ Often the sublattice-update is also called parallel update.
} 
an update. For this situation several results are known [6]-[14]. Especially it can be shown that for random-sequential update the mean-field Ansatz yields the exact equilibrium state [3, 8], which is equivalent to the fact that for a fixed number of cars every arrangement of cars occurs with the same probability. Therefore it is quite natural to take the mean-field approach also as a starting point for the investigation of higher velocities $v_{\max }>1$ and parallel update.

Our main interest will be the calculation of the so-called fundamental diagram (flow $q$ vs. density $\rho=N / L)$. As described in [3] these results can be compared directly with measurements of real traffic [16]. One expects a transition from laminar flow to start-stop waves with increasing car density. For $v_{\max }=1$ it is easy to see that the fundamental diagram is symmetric with respect to $\rho=1 / 2$ due to particle-hole symmetry. This is not true for realistic traffic where on finds a distinct asymmetry where the maximum of the flow is shifted to lower values of $\rho(\sim 0.2)$.

In the simple mean-field theory approach on assumes that two neighbouring sites on the ring are completely uncorrelated. Instead of applying the four update-rules in the order 1-2-3-4 we use the order 2-3-4-1, i.e. we write down the evolution equations of the configurations of the system after the first step. This has the advantage that every site is in one of the $v_{\max }+1$ states $v=0,1, \ldots, v_{\max }$ where a state $v \geq 1$ denotes a car with velocity $v$ and the state 0 denotes an empty site. Note, that there are no cars with velocity zero since after the acceleration step every car has at least velocity 1 . The technical advantage of this procedure lies in the fact that one reduces the number of evolution equations by 1 without changing the result. The changed ordering 2-3-4-1 has to be taken into account in the calculation of the flow $q$. Therefore the complete dynamics of the systems are determined by a set of $v_{\max }$ coupled nonlinear equations for the densities $c_{v}(t)$ of cars with velocity $v$. In general, this time-dependent equations cannot be solved exactly due to the nonlinearities. However, the equilibrium properties in the mean-field limit can be derived exactly for any finite velocity $v_{\max }$ [17] (see Fig. 1 for $v_{\max }=1$ and Fig. 3 for $v_{\max }=2$ ).

In the next step we improve the simple mean-field theory by taking into account neighbour-correlations. In the $n$-site approximation one writes down self-consistent evolution equations for chain segments of length $n$ where neighbouring segments have $n-1$ sites in common?. Here self-consistency means that one has to deal with conditional probabilities for the sites not belonging to the chain segment under consideration. The number of equations is $\left(v_{\max }+1\right)^{n}$. Since a car can drive at most $v_{\max }$ sites in one update-step one should take at least $n=v_{\max }$ to get reasonable results.

For $v_{\max }=1$ and parallel update the $n$-site approximations with $n \geq 2$ become identical showing that the 2-site approximation gives the exact result. This new result comes not surprising since for random-sequential update mean-field (1-site approximation) becomes exact but parallel update rules in general produce stronger

\footnotetext{
${ }^{5}$ This $n$-site approximation is similar to the $(n, n-1)$-cluster approximation of 18 .
} 
correlations. Explicitely we find in the thermodynamic limit for the probabilities $P\left(\sigma_{1}, \sigma_{2}\right)$ to find neighboured sites in the states $\sigma_{1}$ and $\sigma_{2}$ (where $\sigma_{j}=0,1$ and $\sigma_{j}=0$ again denotes an empty site and $\left.\bar{p}=1-p\right)$

$$
\begin{aligned}
P(0,1)=P(1,0) & =\frac{1-\sqrt{1-4 \bar{p} \rho(1-\rho)}}{2 \bar{p}}, \\
P(0,0) & =1-\rho-P(1,0) \\
P(1,1) & =\rho-P(1,0) .
\end{aligned}
$$

The corresponding 'groundstate' for a finite system is given by

$$
\mathcal{P}(N, L)=\frac{1}{\mathcal{N}} \sum_{\{\sigma\}}^{\prime} \prod_{j=1}^{L} P\left(\sigma_{j}, \sigma_{j+1}\right) .
$$

Here $\mathcal{N}$ is a normalization constant and the sum $\sum^{\prime}$ runs over all configurations with a fixed number $N$ of cars (i.e. $\sum_{j=1}^{L} \sigma_{j}=1$ ). This result can be shown to be exact for any finite ring with a fixed number of cars. It is interesting to note that in contrast to random-sequential dynamics parallel dynamics lead to an effective attraction between 'particles' and 'holes' (i.e. $P(0) P(1)=\rho(1-\rho) \leq P(01)$ ) and thus to a higher flow. This flow can obtained from the probabilities (11) and yields the following fundamental diagram (see Fig. 2)

$$
q=(1-p) P(1,0)=\frac{1-\sqrt{1-4 \bar{p} \rho(1-\rho)}}{2} .
$$

For $v_{\max }=2$ we have investigated the $n$-site approximations up to $n=5$. Unfortunately, the approximation seems not to become exact in this case. As can be seen from Fig. 3 the maximum difference between the 4 - and 5 -site approximation is less than 1\%. This suggests that these approximations are already close to the exact result. This assertion is further supported by the excellent agreement with the numerical simulations [17]. As can be seen from Fig. 3 the neighbour correlations lead to an increase of the flux $q$. In addition we like to point out that the fundamental diagram for $v_{\max }=2$ shows the asymmetry known from experimental data [16]. The details of the method and further results will be published elsewhere [17, 19].

Apart from periodic boundary conditions also other types of boundary conditions are relevant for the description of traffic flow. Indeed, it is very important for measurement of real traffic whether the observed situation corresponds to free traffic or to part of a bottleneck where the long-range correlations are stronger [16]. The most natural situation for a bottleneck occurs if one considers the reduction (over a finite length) of two-lane to one-lane traffic due to obstacles on one lane. At the end one has again two-lane-traffic. In principle, this is equivalent to one-lane traffic with given input- and output-rate at the boundaries. From a technical point of view the 
bottleneck situation is - even in mean-field approximation - much more complicated. Whereas in the ring geometry the density is fixed by the initial condition in the bottleneck the system evolves itself to a stationary state with a certain mean density. This can be seen explicitly for $v_{\max }=1$ and random sequential dynamics in [9]-12] where the flux through the bottleneck is independent of the input- and output-rates over a wide range of these parameters. For larger velocities $v_{\max }>1$ the bottleneck situation will be studied in a future publication [19].

We also investigated simple models for two-lane traffic. Here the update rules are not defined by 1-4. Instead we have introduced fixed densities $\rho_{1}$ and $\rho_{2}$ of cars with velocity 1 and 2 , respectively. Surprisingly we find in mean-field approximation that the fundamental diagram of each lane is asymmetric but the maximum is shifted to larger values of $\rho\left(\rho_{\max }>1 / 2\right)$. Further investigations will show if this is an artefact of mean-field theory or an intrinsic property of our model 17].

In conclusion, we have studied simple automaton models for the description of traffic flow. It seems that the equilibriium properties of these types of models can be described accurately by improved mean-field theories ( $n$-site approximations). On the other hand the dynamics of these systems seems to be rather complicated. We like to stress that these models also can be used to describe real physical problems such as asymmetric exclusion processes and surface roughening. We also have applied the improved mean-field theory [19] to the Domany-Kinzel cellular automaton [20] and find a phase diagram which is in very good agreement with recent computer simulation studies [21, 22].

\section{Acknowledgement}

This work has been performed within the research program of the Sonderforschungsbereich 341 (Köln-Aachen-Jülich).

We like to thank N. Ito and K. Nagel for useful discussions. 


\section{References}

[1] Wolfram S 1986 Theory and Applications of Cellular Automata (Singapore: World Scientific)

[2] Frisch U, Hasslacher B and Pomeau Y 1986 Phys. Rev. Lett. 561505

[3] Nagel K and Schreckenberg M 1992 J. Phys. I France 22221

[4] Biham O, Middleton A A and Levine D 1992 Phys. Rev. A 46 R6124

[5] Cuesta J A, Martinez F C, Molera J M and Sánchez A 1993 (preprint)

[6] Kandel D, Domany E and Nienhaus B 1990 J. Phys. A: Math Gen. 23 L755

[7] Janowsky S A and Lebowitz J L 1992 Phys. Rev. A 45618

[8] Gwa L-H and Spohn H 1992 Phys. Rev. A 46844

[9] Derrida B, Domany E and Mukamel D 1992 J. Stat. Phys. 69667

[10] Derrida B and Evans M R 1993 J. Phys. I France 3311

[11] Derrida B, Evans M R, Hakim V and Pasquier V 1993 J. Phys. A: Math Gen. 26, 1493

[12] Schütz G and Domany E 1993 (preprint)

[13] Schütz G 1993 (preprint)

[14] Schütz G 1993 J. Stat. Phys. 71471

[15] Tang L-H, Forrest B M and Wolf D E 1992 Phys. Rev. A 457162

[16] Hall F L, Brian L A and Gunter M A 1986 Transpn. Res. A 20A 197

[17] Ito N, Nagel K, Schadschneider A and Schreckenberg M 1993 (to be published)

[18] ben-Avraham D and Köhler J 1992 Phys. Rev. B 458358

[19] Schadschneider A and Schreckenberg M 1993 (in preparation)

[20] Domany E and Kinzel W 1984 Phys. Rev. Lett. 53311

Kinzel W 1985 Z. Phys. B 58229

[21] Martins M L, Verona de Resende H F, Tsallis C and de Magalhães A C N 1991 Phys. Rev. Lett. 662045

[22] Kohring G and Schreckenberg M 1992 J. Phys. I France 22033 


\section{Figure Captions}

Fig. 1: Fundamental diagram as obtained from the mean-field theory (dotted line) and from the 2-site approximation for $v_{\max }=1$ and $p=\bar{p}=1 / 2$. Note that the 2 -site approximation already gives the exact result.

Fig. 2: Exact fundamental diagram for $v_{\max }=1$ and different values of $p$.

Fig. 3: Fundamental diagram for $v_{\max }=2$ and $p=1 / 2$ as obtained from the 1-site approximation (lowest curve) up to the 5-site approximation (highest curve). 